\title{
Bilateral common carotid artery ultrasound for prediction of incident strokes using intima-media thickness and external diameter: an observational study
}

Marsha L Eigenbrodt ${ }^{*}$, Gregory W Evans ${ }^{2}$, Kathryn M Rose ${ }^{3,4}$, Zoran Bursac ${ }^{5}$, Richard E Tracy ${ }^{6}$, Jawahar L Mehta ${ }^{7}$ and David J Couper ${ }^{8}$

\begin{abstract}
Background: External common carotid artery (CCA) diameter and intima-media thickness (IMT) are independently associated with incident stroke and other cardiovascular events. Arterial geometry such as large IMT and large diameter may reflect vulnerable plaques and so impact stroke risk. Finally, arterial changes that exist bilaterally may increase stroke risk.

Method: We studied middle-aged men and women $(n=7276)$ from a prospective observational study who had right (R) and left (L) CCA IMT and external diameters measured via B-mode ultrasound (1987-89) in order to categorize CCA geometry. Using side- and gender-specific IMT and diameter medians, we categorized each measurement as large ( $\geq$ median) vs. not large ( $<$ median) and defined four geometries: both IMT and diameter were large, only one parameter was large, or neither was large (reference group). Participants were followed for first time stroke through December 31, 1999. We used proportional hazards models to assess associations between right and left CCA geometries with new stroke. We also calculated positive and negative likelihood ratios (+LR and -LR) for CCA bilateral phenotypes as a measure of diagnostic accuracy.
\end{abstract}

Results: Presence of both large CCA IMT and large diameter on one side was associated with strong stroke risk even after risk factor adjustment (men: RCCA hazard ratio $[\mathrm{HR}]=3.7$ 95\% confidence interval $[\mathrm{Cl}]=1.9-7.4$; LCCA $H R=2.495 \% \mathrm{Cl}=1.4-4.4$; women: RCCA HR=4.0 95\% Cl=1.5-10.5; LCCA HR=5.7 95\% Cl=1.7-19.0). Presence of both large IMT and large diameter bilaterally was the strongest predictor of stroke identifying $64 \%$ of women and $44 \%$ of men who developed strokes. This phenotype showed potential for predicting stroke among individuals (women: $+\mathrm{LR}=3.1,95 \% \mathrm{Cl}=2.6-3.8$; men: $+\mathrm{LR}=2.3,95 \% \mathrm{Cl}=1.8-2.8$ ).

Conclusion: Bilateral carotid artery geometries may be useful for stroke risk prediction.

Keywords: Stroke, Atherosclerosis, Carotid arteries, Ultrasound, Intima-media thickness, Arterial diameter, Arterial geometry, Bilateral, Prospective study

\footnotetext{
* Correspondence: meigenbrodt@gmail.com

${ }^{1}$ College of Medicine and Fay W. Boozman College of Public Health,

University of Arkansas for Medical Sciences, Little Rock, AR, USA

Full list of author information is available at the end of the article
} 


\section{Background}

Stroke is a leading cause of death and adult disability in the US [1,2]. About $88 \%$ of strokes in Western countries are ischemic [3] with almost $50 \%$ of strokes occurring in the presence of atherosclerosis of the extracranial or large intracranial arteries [4]. Significant stenosis of large arteries accounts for only $20 \%$ of ischemic strokes overall [3] and only 10\% in asymptomatic people [4]. Vulnerable plaques, which may rupture to produce strokes [4], are frequently found in arteries with less than 50\% lumen stenosis [5] and have been associated with ispsilateral cryptogenic strokes [6]. So, markers for stroke other than stenosis are needed [7].

Models for predicting overall cardiovascular disease (CVD) risk [8-10] and stroke-specific risk [11,12] are generally acceptable, but do have limitations $[8,13,14]$ such as limited risk factor measurements, and assuming risk factor effects are consistent across ages and in different risk factor groups. In particular, better stratification models for women and others with low shortterm, but high lifetime risk are needed [13,15]. Direct vascular examination has been proposed as a way to improve stroke prediction in individuals $[7,16]$. Modest improvements in prediction were gained by adding various carotid IMT measures to CVD $[17,18]$ and stroke $[11,17]$ prediction models. Though not tested in the stroke prediction model, carotid stiffness measures derived from carotid diameters were independently associated with stroke [19]. A promising, but expensive method is high resolution MRI [7] which can identify carotid plaque characteristics that increase a person's stroke risk [5,7]. Hypertension, the most powerful predictor of both lacunar and cortical infarcts [20] has been associated with increased elastic arterial diameters, as has metabolic syndrome and other risk factors $[21,22]$. Likewise, outward remodeling occurs more frequently in areas of vulnerable plaques [23]. So, arterial diameter enlargement could help identify stroke risk as suggested in a case control study where carotid diameters were larger in stroke than in non-stroke patients [24]. In fact, a recent prospective study found that both intima-media thickness (IMT) and external common carotid artery (CCA) diameter were independently associated with incident stroke and that diameter added discrimination [25]. However, external artery diameter may reflect different underlying mechanisms/characteristics depending upon the coexisting wall thickness [26,27]. Thus, we evaluated four CCA geometries on the right and left sides defined by B-mode ultrasound assessment of both IMT and external diameter, and then we explored bilateral combinations of these arterial patterns as phenotypes for risk discrimination.

\section{Subjects and methods}

Sample: The Atherosclerosis Risk in Community (ARIC) Study is a prospective study of 15792 men and women who were 45-64 years of age at baseline (1987-89). Participants are predominantly black and white and were recruited from four U. S. communities [28]. All participants signed informed consents. Committees for human subjects' protection at each participating site approved the original ARIC study, and the current ancillary study was approved at the University of Arkansas for Medical Sciences. NHLBI provided the ARIC limited access data (ARICLAD) for this study. The ARICLAD contains $99.6 \%(n=15732)$ of the individuals from the full ARIC study. Not all participants had usable right and left CCA measurements of IMT and external diameter (diameter), and because the missing LCCA and RCCA parameters overlapped only partially, complete CCA IMT and diameter measures were available for only 10096 participants. After removing persons with missing computer algorithmdefined stroke at baseline, 7874 persons remained. Additional restrictions for missing information on baseline prevalent coronary heart $(\mathrm{n}=138)$, and other major potential confounders [age, sex, race, diabetes mellitus, systolic and diastolic blood pressure, current drinking or smoking status, low and high density lipoproteins (LDL and HDL), fibrinogen, glucose, white blood count, triglyceride, and hypertension status $(n=299)]$ left 7437 in the baseline sample for assessing risk factor associations with right and left arterial geometries. For analyses of new strokes, the 161 persons with stroke at baseline were removed, leaving 7276 individuals for incidence analyses.

\section{Outcome: stroke definitions}

The baseline definition of stroke that we used was ARIC's computer algorithm definition based on six symptoms (speech, vision, double vision, numbness, paralysis and dizziness) [29]. Incident ischemic stroke events were identified from information collected during annual telephone interviews and from an ongoing community surveillance program and were validated and classified as ischemic vs. hemorrhagic using hospital and autopsy information [30]. The current study evaluates definite or probable ischemic strokes that include both thrombotic and cardio-embolic events that lasted more than 24 hours and which were not secondary to trauma, neoplasms, infections, vasculitis, or hematologic abnormalities.

\section{Main exposures}

The main exposures, unilateral CCA geometric patterns and bilateral phenotypes, were defined based on ultrasound measurements of CCA IMT and external diameter (interadventitial distance) at baseline. The ARIC 
procedure for CCA ultrasound measurement has been previously described [21,31-33]. Briefly, ultrasound scans of three carotid artery segments from the right and the left were recorded for later reading according to a standardized protocol. All images were read at a central reading center using frames with the largest lumen (indicating systole) that had optimal arterial wall boundaries [33]. The right and left CCA far wall IMT and external diameter measures used in this study were the means of multiple measurements attempted at $1 \mathrm{~mm}$ intervals from the $1 \mathrm{~cm}$ carotid segment just proximal to the carotid bifurcation using standard interfaces and optimal angle. We used gender-specific medians of the RCCA and LCCA IMTs and external diameters from our study sample (Table 1) to categorize each parameter as large (upper $50^{\text {th }}$ percentile) or not large (lower $50^{\text {th }}$ percentile) to achieve comparably sized groups for comparisons of IMT and diameter effects. Then, four geometric patterns were defined for each CCA (IMT and diameter both large, only IMT large, only diameter large, and neither large $=$ reference). Cross classification of $R$ and L CCA patterns produced 16 categories that could be reduced to ten non-overlapping categories/phenotypes which might have different implications. For instance, we hypothesized that large diameter and large IMT on the same side may indicate vulnerable plaques, which if present bilaterally would indicate a high risk group. Large IMT in the absence of large diameter may indicate stable plaques that narrow the lumen. Vascular narrowing on both sides could produce cerebral ischemia in the presence of hypotensive episodes. Bilaterally large diameter in the absence of large IMT could indicate the presence of known factors such as hypertension [21,22] that also cause stroke. Unilateral abnormalities could indicate only focal change, or less definitive abnormalities. After

\begin{tabular}{|c|c|c|}
\hline Gender & Vascular parameter & Median \\
\hline Men $(n=3330)$ & & $\mathrm{mm}$ \\
\hline \multirow[t]{2}{*}{ RCCA } & IMT & 0.663 \\
\hline & Diameter & 8.092 \\
\hline \multirow[t]{2}{*}{ LCCA } & IMT & 0.676 \\
\hline & Diameter & 7.997 \\
\hline \multicolumn{3}{|c|}{ Women $(n=4107)$} \\
\hline \multirow[t]{2}{*}{ RCCA } & IMT & 0.609 \\
\hline & Diameter & 7.303 \\
\hline \multirow[t]{2}{*}{ LCCA } & IMT & 0.603 \\
\hline & Diameter & 7.199 \\
\hline
\end{tabular}

*Determined by B-mode ultrasound.

RCCA and LCCA = right and left common carotid artery respectively. IMT intima-media thickness. assessing bilateral phenotypes, we then explored various combinations of these phenotypes as stroke predictors.

\section{Other variables}

Baseline plaques were identified from any right or left carotid segment (common, internal, and bifurcation) [31,32]. The methods for defining smoking, drinking and disease status, as well as methods for laboratory measures and for anthropometric measures have been summarized previously [11,32]. Diabetes mellitus was defined as a fasting glucose level $\geq 126 \mathrm{mg} / \mathrm{dl}(7.0 \mathrm{mmol} / \mathrm{L})$, a nonfasting level $\geq 200 \mathrm{mg} / \mathrm{dl}(11.1 \mathrm{mmol} / \mathrm{L})$ (hexokinase method), self-reported diagnosis of diabetes, or use of medication for diabetes. Baseline, sitting, systolic and diastolic blood pressures were the means of the second and third blood pressure values obtained using a standard sphygmomanometer. Hypertension was defined as blood pressure $\geq 140 / 90 \mathrm{~mm} \mathrm{Hg}$ or use of anti-hypertensive medication within 2 weeks of the clinic visit. Smoking status and drinking status were self-reported. We used the ARIC definition of coronary heart disease (CHD) which includes myocardial infarction from the baseline electrocardiogram, a self-reported history of a myocardial infarction or of specific types of heart vascular surgery. Body mass index (BMI, weight in $\mathrm{kg} /$ height in $\mathrm{m}^{2}$ ) was calculated from baseline measurements. Central laboratories measured fibrinogen, total cholesterol, high density lipoprotein cholesterol and triglycerides, while low density lipoprotein cholesterol was computed. Local laboratories measured white blood counts.

\section{Statistical methods}

Analyses were performed using SAS 9.1 except for diagnostic test evaluations which were performed using online software [34]. Because of the potential for gender differences in external diameter associations [35,36], we evaluated men and women separately. The Kruskal Wallis and chi-square tests were used to assess overall significance of variations in baseline characteristics across the arterial geometries. Post hoc comparisons were made using the Student's t test and chi-square tests. Gender- and side-specific Cox-proportional hazards models [37] were used to assess the relationship between the LCCA and RCCA geometric patterns with incident ischemic stroke. Basic models included indicator variables for CCA geometries and adjusted for age, race, and standing height and were followed by multivariable models that retained covariates based on significance $(p<0.05)$ and confounding ( $10 \%$ change in the point estimate for any of three indicator variables for CCA geometries). Finally, stroke incidence, sensitivity, specificity, and unadjusted relative risks were evaluated for the bilateral arterial phenotypes formed from cross-classification 
of the right and left CCA geometric patterns. To assess their utility as indicators of stroke risk, positive and negative likelihood ratios (+LR and -LR respectively) and 95\% confidence intervals $(\mathrm{CI})$ were calculated using online software [34] for several phenotypes in our study and from numbers calculated from published stroke incidence and sensitivities and specificities for the top quintile of the Framingham general CVD risk scores and cerebrovascular risk scores [12].

\section{Results}

Median values for IMT and diameter were slightly larger for men than women (Table 1). The percentages of persons categorized as having specific geometries were very similar on the right and left (Table 2). However, for individuals, the right and left geometries were often discrepant with the Kappa statistics indicating only fair agreement in CCA classification on the right and left.

Table 3 provides the baseline characteristics for persons exhibiting the side-specific arterial geometries. Persons who exhibited both large IMT and large diameter on the right or left tended to have the most detrimental collection of characteristics: a larger percentage had carotid lesions (plaques), hypertension, and diabetes

\begin{tabular}{|c|c|c|c|}
\hline \multirow{2}{*}{$\begin{array}{l}\text { Large } \\
\text { parameter(s) }\end{array}$} & \multicolumn{2}{|c|}{ Number (\%) } & \multirow{2}{*}{$\begin{array}{l}\% \text { Observed right-left } \\
\text { Agreement }^{*}(95 \% \mathrm{Cl})\end{array}$} \\
\hline & RCCA & LCCA & \\
\hline \multicolumn{4}{|l|}{ Men $(\mathrm{N}=3330)$} \\
\hline IMT and diameter & 1068 (32.1) & $1074(32.3)$ & $41.8(39.2-44.3)$ \\
\hline IMT only & $599(18.0)$ & $607(18.2)$ & $23.3(20.7-26.1)$ \\
\hline Diameter only & $598(18.0)$ & $599(18.0)$ & $23.7(21.1-26.5)$ \\
\hline Neither & 1065 (32.0) & 1050 (31.5) & $46.3(43.7-48.9)$ \\
\hline \multicolumn{4}{|l|}{ Women ( $N=4107$ ) } \\
\hline IMT and diameter & 1307 (31.82) & 1391 (33.9) & $43.3(40.9-45.6)$ \\
\hline IMT only & 751 (18.29) & 795 (19.4) & $24.7(22.3-27.4)$ \\
\hline Diameter only & 755 (18.4) & $668(16.3)$ & $26.6(24.2-29.2)$ \\
\hline Neither & 1294 (31.5) & $1253(30.5)$ & $47.9(45.6-50.2)$ \\
\hline \multicolumn{4}{|l|}{ All (N=7437) } \\
\hline IMT and diameter & 2375 (31.9) & 2465 (33.2) & $42.6(40.9-44.3)$ \\
\hline IMT only & $1350(18.2)$ & 1402 (18.9) & $24.1(22.6-26.0)$ \\
\hline Diameter only & $1353(18.2)$ & $1267(17.0)$ & $25.3(23.5-27.2)$ \\
\hline Neither & 2359 (31.7) & 2303 (31.0) & $47.2(45.5-48.9)$ \\
\hline
\end{tabular}

Women: Kappa $=0.38,95 \% \mathrm{Cl} 0.36-0.40$; Men Kappa $=0.35,95 \% \mathrm{Cl}$ 0.33-0.38; All: Kappa $=0.37,95 \% \mathrm{Cl}=0.35-0.38$ )

*\% having the characteristic in both the RCCA and LCCA if it is present on either side.

${ }^{\dagger}$ RCCA and LCCA (right and left common carotid artery) geometries are based on ultrasound measurements of IMT and diameters at baseline where large is defined as $\geq$ the gender- and side-specific medians for IMT and external diameter in Table 1. mellitus and higher levels of many other detrimental risk factors, such as systolic blood pressure, than were found among persons without both large IMT and diameter. Women with this pattern also had much higher CHD prevalence than women with other arterial geometries. For men, CHD prevalence was similar when both IMT and diameter was large as when only IMT was large. Risk factors were also elevated in men and women with isolated large IMT or isolated large diameter compared to the respective reference group. For men, stroke prevalence did not vary by CCA geometry, but for women, stroke prevalence was higher when the LCCA diameter was large compared to the LCCA reference.

Between baseline (1987-1989) and the end of December 1999, 181 new strokes were documented, 66 among women and 115 among men (1.6\% and 3.5\% respectively). The stroke risks associated with separate RCCA and LCCA geometries are shown in Figure 1 Adjusting for age, race, and height, men and women having both large IMT and large diameter in one CCA at baseline had a strong risk of developing a stroke relative to the respective reference group and the risk remained large and statistically significant after risk factor adjustment. Also, while not statistically different, the hazard ratios were consistently larger than what was found for persons having isolated large IMT or diameter on the respective side.

Cross-classification of RCCA and LCCA geometries allowed evaluation of bilateral geometric CCA phenotypes (Table 4). Twenty percent of men and $21 \%$ of women had both large IMT and large diameter in the right and left CCA while almost 19\% of men and women had neither large IMT nor large diameter on either side (reference). Considering the non-overlapping phenotypes in Table 4, the highest stroke cumulative incidence $(7.7 \%$ for men and $4.9 \%$ for women) occurred in those with both IMT and diameter large bilaterally and low incidence was found in the reference groups where neither IMT nor diameter was large producing very large relative risks (women $R R=16.3$; men $R R=6.4$ ). In the absence of at least one bilaterally large parameter, stroke incidence was generally low.

Table 5 presents diagnostic test information for selected bilateral arterial phenotypes. For the phenotype where CCA IMT and diameter were both large bilaterally, the sensitivity was $64 \%$ for women and $44 \%$ for men while specificity was about $80 \%$ for men and women. This resulted in +LRs of over 2 for men and approximately 3 for women. Expanding the positive test to include persons with any component large bilaterally (large IMT bilaterally and/or large diameter bilaterally) resulted in sensitivities greater than $80 \%$ for both men and women with improved -LRs that were comparable to those calculated for either Framingham 
Table 3 Gender-specific percentages and means of baseline characteristics for right and left common carotid artery geometries

\begin{tabular}{|c|c|c|c|c|c|c|c|c|}
\hline \multirow[b]{5}{*}{ Women } & \multicolumn{4}{|c|}{ Mean \pm SD or $n$ (\%) for } & \multicolumn{4}{|c|}{ Mean $\pm S D$ or $n(\%)$ for } \\
\hline & \multicolumn{4}{|c|}{ RCCA Geometries defined by large* component(s): } & \multicolumn{4}{|c|}{ LCCA Geometries defined by large* component(s): } \\
\hline & $\mathrm{IMT}+$ & IMT & Diameter & Neither & $\mathrm{IMT}+$ & IMT & Diameter & Neither \\
\hline & Diameter & Only & Only & & Diameter & Only & Only & \\
\hline & $\mathrm{N}=1307$ & $\mathrm{~N}=751$ & $\mathrm{~N}=755$ & $\mathrm{~N}=1294$ & $\mathrm{~N}=1391$ & $\mathrm{~N}=795$ & $\mathrm{~N}=668$ & $\mathrm{~N}=1253$ \\
\hline Age, y & $55.9 \pm 5.5^{\dagger}$ & $53.9 \pm 5.5^{\dagger}$ & $53.5 \pm 5.6^{\dagger}$ & $51.4 \pm 5.2$ & $55.9 \pm 5.6^{\dagger}$ & $53.8 \pm 5.6^{\dagger}$ & $53.3 \pm 5.5^{\dagger}$ & $51.5 \pm 5.2$ \\
\hline Race, black & $507(38.8)^{\dagger}$ & $226(30.1)^{\dagger}$ & $224(29.7)^{\dagger}$ & $262(20.2)$ & $507(36.4)^{\dagger}$ & $197(24.8)$ & $246(36.8)^{\dagger}$ & $269(21.5)$ \\
\hline $\mathrm{CHD}$ & $43(3.3)^{\dagger}$ & $13(1.7)^{\S}$ & $8(1.1)$ & $7(0.5)$ & $41(2.9)^{\dagger}$ & $11(1.4)$ & $11(1.6)^{\S}$ & $8(0.6)$ \\
\hline Carotid lesions & $554(46.9)^{\dagger}$ & $227(34.6)^{\dagger}$ & $193(27.8)^{\S}$ & $279(23.7)$ & $548(44.0)^{\dagger}$ & $241(33.8)^{\dagger}$ & $178(29.1)$ & $286(25.1)$ \\
\hline Hypertension & $634(48.5)^{\dagger}$ & $199(26.5)^{\dagger}$ & $252(33.4)^{\dagger}$ & $236(18.2)$ & $659(47.4)^{\dagger}$ & $207(26.0)^{\dagger}$ & $229(34.3)^{\dagger}$ & $226(18.0)$ \\
\hline Diabetes mellitus & $202(15.5)^{\dagger}$ & $64(8.5)^{\dagger}$ & $50(6.6)^{\S}$ & $58(4.5)$ & $194(13.9)^{\dagger}$ & $67(8.4)^{\dagger}$ & $56(8.4)^{\ddagger}$ & $57(4.5)$ \\
\hline Current smoker & $357(27.3)$ & $164(21.8)$ & $230(30.5)^{\ddagger}$ & $319(24.7)$ & $391(28.1)^{\S}$ & $194(24.4)$ & $191(28.6)^{\S}$ & $294(23.5)$ \\
\hline Current drink & $596(45.6)^{\dagger}$ & $394(52.5)^{\dagger}$ & $381(50.5)^{\dagger}$ & $794(61.4)$ & $654(47.0)^{\dagger}$ & $436(54.8)$ & $334(50.0)^{\dagger}$ & $741(59.1)$ \\
\hline Systolic BP mm Hg & $128 \pm 22^{\dagger}$ & $116 \pm 16^{\dagger}$ & $121 \pm 19^{\dagger}$ & $112 \pm 16$ & $128 \pm 22^{\dagger}$ & $116 \pm 15^{\dagger}$ & $121 \pm 19^{\dagger}$ & $113 \pm 16$ \\
\hline Diastolic BP mm Hg & $73 \pm 12^{+}$ & $71 \pm 10^{\S}$ & $72 \pm 11^{\dagger}$ & $70 \pm 10$ & $73 \pm 12^{+}$ & $71 \pm 10^{\S}$ & $73 \pm 11^{\dagger}$ & $70 \pm 10$ \\
\hline Height, cm & $163.0 \pm 5.9^{\ddagger}$ & $162.0 \pm 6.0$ & $163 \pm 6.0^{\S}$ & $162.3 \pm 5.7$ & $162.9 \pm 6.0^{\ddagger}$ & $161.9 \pm 5.8$ & $163.1 \pm 5.9^{\ddagger}$ & $162.3 \pm 5.8$ \\
\hline $\mathrm{BMI}, \mathrm{kg} / \mathrm{m}^{2}$ & $28.0 \pm 5.7^{\dagger}$ & $26.5 \pm 5.0^{\dagger}$ & $27.3 \pm 5.8^{\dagger}$ & $25.5 \pm 4.7$ & $27.8 \pm 5.6^{\dagger}$ & $26.5 \pm 5.1^{\dagger}$ & $27.5 \pm 5.9^{\dagger}$ & $25.5 \pm 4.7$ \\
\hline Glucose, IU & $6.32 \pm 2.94^{\dagger}$ & $5.76 \pm 1.92^{\ddagger}$ & $5.69 \pm 1.65^{\S}$ & $5.51 \pm 1.27$ & $6.21 \pm 2.76^{\dagger}$ & $5.80 \pm 1.90^{\dagger}$ & $5.80 \pm 2.05^{\dagger}$ & $5.49 \pm 1.28$ \\
\hline HDL, IU & $1.46 \pm 0.43^{\dagger}$ & $1.55 \pm 0.45$ & $1.50 \pm 0.47^{\ddagger}$ & $1.57 \pm 0.45$ & $1.45 \pm 0.44^{\dagger}$ & $1.54 \pm 0.45$ & $1.54 \pm 0.47$ & $1.57 \pm 0.43$ \\
\hline LDL, IU & $3.65 \pm 1.05^{\dagger}$ & $3.56 \pm 1.05^{\dagger}$ & $3.41 \pm 1.06^{\ddagger}$ & $3.27 \pm 1.03$ & $3.65 \pm 1.06^{\dagger}$ & $3.54 \pm 1.04^{\dagger}$ & $3.39 \pm 1.10^{\S}$ & $3.27 \pm 0.99$ \\
\hline Fibrinogen, mg/dL & $314 \pm 71^{\dagger}$ & $298 \pm 56$ & $308 \pm 64^{\dagger}$ & $293 \pm 57$ & $315 \pm 69^{\dagger}$ & $299 \pm 61^{\S}$ & $303 \pm 62^{\dagger}$ & $293 \pm 57$ \\
\hline $\mathrm{WBC}, 1000 \mathrm{~s} / \mathrm{mm}^{3}$ & $6.00 \pm 1.86$ & $5.72 \pm 1.76$ & $6.01 \pm 1.92$ & $5.86 \pm 1.79$ & $6.00 \pm 1.88^{\ddagger}$ & $5.94 \pm 1.81$ & $5.89 \pm 1.87$ & $5.80 \pm 1.77$ \\
\hline Triglyceride, IU & $1.35 \pm 0.67^{\dagger}$ & $1.28 \pm 0.65^{\dagger}$ & $1.27 \pm 0.63^{\ddagger}$ & $1.18 \pm 0.60$ & $1.35 \pm 0.67^{\dagger}$ & $1.29 \pm 0.64^{\dagger}$ & $1.24 \pm 0.64^{\S}$ & $1.18 \pm 0.59$ \\
\hline Prevalent stroke & $37(2.8)$ & $11(1.5)$ & $20(2.6)$ & $25(1.9)$ & $32(2.3)$ & $10(1.3)$ & $28(4.2)^{\ddagger}$ & $23(1.8)$ \\
\hline MEN & $\mathrm{N}=1068$ & $\mathrm{~N}=599$ & $\mathrm{~N}=598$ & $\mathrm{~N}=1065$ & $\mathrm{~N}=1074$ & $\mathrm{~N}=607$ & $\mathrm{~N}=599$ & $\mathrm{~N}=1050$ \\
\hline Age, y & $56.5 \pm 5.5^{\dagger}$ & $55.1 \pm 5.6^{\dagger}$ & $53.7 \pm 5.8^{\dagger}$ & $52.4 \pm 5.4$ & $56.4 \pm 5.5^{\dagger}$ & $54.6 \pm 5.6^{\dagger}$ & $53.9 \pm 5.7^{\dagger}$ & $52.6 \pm 5.5$ \\
\hline Race, black & $311(29.1)^{\dagger}$ & $171(28.5)^{\dagger}$ & $131(21.9)$ & $218(20.5)$ & $294(27.4)^{\ddagger}$ & $147(24.2)$ & $160(26.7)^{\S}$ & $230(21.9)$ \\
\hline CHD & $100(9.4)^{\dagger}$ & $59(9.8)^{\dagger}$ & $38(6.4)$ & $56(5.3)$ & $111(10.3)^{\dagger}$ & $58(9.6)^{\dagger}$ & $36(6.0)$ & $48(4.6)$ \\
\hline Carotid lesions & $551(58.7)^{\dagger}$ & $264(50.8)^{\dagger}$ & $231(43.3)^{\dagger}$ & $324(34.1)$ & $546(57.9)^{\dagger}$ & $275(52.0)^{\dagger}$ & $216(40.5)$ & $333(35.5)$ \\
\hline Hypertension & $466(43.6)^{\dagger}$ & $174(29.0)^{\ddagger}$ & $214(35.8)^{\dagger}$ & $235(22.1)$ & $470(43.8)^{\dagger}$ & $184(30.3)^{\dagger}$ & $208(34.7)^{\dagger}$ & 227 (21.6) \\
\hline Diabetes mellitus & $153(14.3)^{\dagger}$ & $76(12.7)^{\dagger}$ & $56(9.4)^{\S}$ & $67(6.3)$ & $152(14.2)^{\dagger}$ & $75(12.4)^{\dagger}$ & $61(10.2)^{\ddagger}$ & $64(6.1)$ \\
\hline
\end{tabular}


Table 3 Gender-specific percentages and means of baseline characteristics for right and left common carotid artery geometries (Continued)

\begin{tabular}{|c|c|c|c|c|c|c|c|c|}
\hline Current smoker & $344(32.2)^{\dagger}$ & $156(26.0)$ & $207(34.6)^{\dagger}$ & $263(24.7)$ & $361(33.6)^{\dagger}$ & $157(25.9)$ & $187(31.2)^{\S}$ & $265(25.2)$ \\
\hline Current drink & $667(62.5)$ & $389(64.9)$ & $408(68.2)$ & $705(66.2)$ & $672(62.6)^{\S}$ & $395(65.1)$ & $398(66.4)$ & $704(67.0)$ \\
\hline Systolic BP, mm Hg & $129 \pm 20^{\dagger}$ & $119 \pm 16^{\ddagger}$ & $123 \pm 19^{\dagger}$ & $116 \pm 14$ & $128 \pm 21^{\dagger}$ & $120 \pm 16^{\dagger}$ & $124 \pm 19^{\dagger}$ & $116 \pm 14$ \\
\hline Diastolic BP, mmHg & $77 \pm 13^{+}$ & $74 \pm 11$ & $76 \pm 12^{\S}$ & $74 \pm 10$ & $76 \pm 13^{+}$ & $75 \pm 11$ & $76 \pm 12^{+}$ & $74 \pm 10$ \\
\hline Height, cm & $176.6 \pm 6.4^{\S}$ & $175.7 \pm 6.5$ & $177.5 \pm 6.4^{\dagger}$ & $176.0 \pm 6.3$ & $176.6 \pm 6.4^{\ddagger}$ & $175.5 \pm 6.3$ & $177.7 \pm 6.4^{\dagger}$ & $175.8 \pm 6.4$ \\
\hline BMl, kg/m² & $27.6 \pm 4.1^{\dagger}$ & $26.7 \pm 3.8^{\S}$ & $26.9 \pm 3.9^{\ddagger}$ & $26.3 \pm 3.5$ & $27.5 \pm 4.0^{\dagger}$ & $26.8 \pm 3.7^{\S}$ & $27.0 \pm 3.9^{\dagger}$ & $26.4 \pm 3.6$ \\
\hline Glucose, IU & $6.28 \pm 2.39^{\dagger}$ & $6.21 \pm 2.34^{\dagger}$ & $5.92 \pm 1.66^{\S}$ & $5.74 \pm 1.09$ & $6.25 \pm 2.34^{\dagger}$ & $6.16 \pm 2.20^{\dagger}$ & $5.98 \pm 1.77^{\S}$ & $5.75 \pm 1.23$ \\
\hline $\mathrm{HDL}, \mathrm{IU}$ & $1.17 \pm 0.37$ & $1.16 \pm 0.34$ & $1.21 \pm 0.43^{\S}$ & $1.16 \pm 0.34$ & $1.17 \pm 0.37$ & $1.13 \pm 0.32^{\ddagger}$ & $1.22 \pm 0.45^{\S}$ & $1.17 \pm 0.34$ \\
\hline LDL, IU & $3.65 \pm 0.95^{\S}$ & $3.71 \pm 0.97^{\dagger}$ & $3.48 \pm 0.96$ & $3.54 \pm 0.94$ & $3.65 \pm 0.98^{\ddagger}$ & $3.70 \pm 0.95^{\dagger}$ & $3.52 \pm 0.96$ & $3.53 \pm 0.92$ \\
\hline Fibrinogen, mg/dL & $305 \pm 67^{\dagger}$ & $294 \pm 62^{\S}$ & $295 \pm 61^{\S}$ & $287 \pm 62$ & $303 \pm 66^{\dagger}$ & $294 \pm 63^{\S}$ & $298 \pm 64^{\ddagger}$ & $287 \pm 61$ \\
\hline WBC, $1000 \mathrm{~s} / \mathrm{mm}^{3}$ & $6.38 \pm 1.98^{\dagger}$ & $6.03 \pm 1.82$ & $6.34 \pm 2.07^{\S}$ & $6.07 \pm 1.83$ & $6.47 \pm 2.06^{\dagger}$ & $6.19 \pm 1.98$ & $6.07 \pm 1.83$ & $6.04 \pm 1.79$ \\
\hline Triglyceride, IU & $1.49 \pm 0.75^{\S}$ & $1.45 \pm 0.74$ & $1.47 \pm 0.78$ & $1.40 \pm 0.72$ & $1.51 \pm 0.78^{\dagger}$ & $1.49 \pm 0.76^{\ddagger}$ & $1.43 \pm 0.72$ & $1.37 \pm 0.72$ \\
\hline Prevalent stroke & $26(2.4)$ & $13(2.2)$ & $12(2.0)$ & $17(1.6))$ & $21(2.0)$ & $15(2.5)$ & $13(2.2)$ & $19(1.8)$ \\
\hline
\end{tabular}

$\mathrm{N}=$ the maximum number possible; some variables have lower numbers available. Fewer persons had carotid plaque information

*Large is defined as $\geq$ the gender- and side-specific medians for IMT and external diameter in Table 1 . 


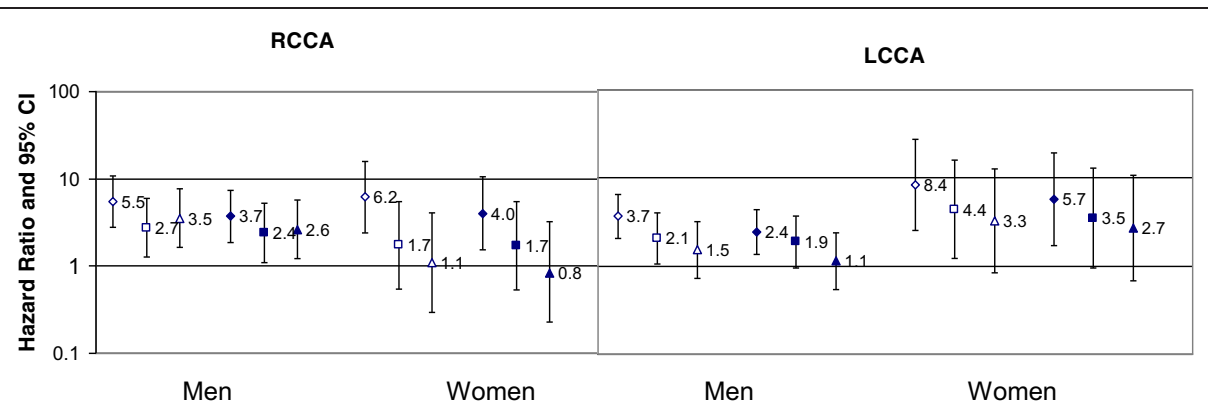

Figure $1 \mathrm{Gender-specific} \mathrm{hazard} \mathrm{ratios} \mathrm{and} 95 \%$ confidence intervals $(\mathrm{CI})$ for first incident ischemic stroke according to baseline common carotid artery (CCA) geometries of the right (RCCA) and left (LCCA) relative to persons with neither large IMT nor large external diameter on the respective side, ARICLAD 1987-1999. Legend: Baseline gender- and side-specific median values for CCA IMT and external diameter were used to categorize each measure as large ( $\geq$ median) or not large ( $<$ median). For each side, diamonds indicate both IMT and diameter are large; squares indicate only IMT is large; triangles indicate only external diameter is large, and the reference group is neither IMT nor diameter is large. Open symbols indicate height, age, and race adjustment, while solid symbols indicate risk factor adjusted risks (covariates for men: baseline age, glucose, diastolic blood pressure, prevalent CHD, hypertension and current smoking; covariates for women RCCA: age, race, prevalent CHD, carotid plaques, triglycerides and its squared term, peripheral white blood count, and systolic blood pressure; LCCA model added body mass index and diabetes mellitus).

scores. However, the reduced specificity produced + LRs that were not as good. The combined phenotype with the best sensitivity was any large IMT (large IMT unilateral or bilateral), but the low specificity resulted in lower $+\mathrm{LR}$ than the phenotype of large IMT and diameter bilaterally.

\section{Discussion}

Most strokes, like other CVD, are caused by multiple factors that lead to atherosclerosis and/or small artery disease [2]. Current CVD risk stratification tools for general populations have limited success in persons with low short-term risk, but high lifetime risk [14] which

Table 4 Incident strokes* for men and women with baseline bilateral common carotid artery phenotypes

\begin{tabular}{|c|c|c|c|c|}
\hline \multirow{2}{*}{$\begin{array}{l}\text { CCA Phenotypes: } \\
\text { Ten non-overlapping phenotypes }\end{array}$} & \multicolumn{2}{|c|}{ Number } & \multicolumn{2}{|c|}{ Incident stroke } \\
\hline & Women & Men & Women N (\%) & Men N (\%) \\
\hline 1) IMT and diameter both large bilaterally & 852 & 662 & $42(4.9)$ & $51(7.7)$ \\
\hline 2) IMT and diameter large on one side and opposite IMT large (bilaterally large IMT) & 271 & 229 & $4(1.5)$ & $12(5.2)$ \\
\hline 3) IMT and diameter large on one side and opposite diameter large (bilaterally large diameter) & 448 & 370 & $6(1.3)$ & $19(5.1)$ \\
\hline 4) IMT and diameter large on one side and neither opposite IMT nor diameter large & 206 & 172 & $1(0.5)$ & $1(0.6)$ \\
\hline 5) Bilaterally large IMT, no large diameter & 322 & 223 & $6(1.9)$ & $10(4.5)$ \\
\hline 6) Unilaterally large IMT ( $R$ or L) and opposite diameter large & 106 & 86 & $1(0.9)$ & 0 \\
\hline 7) Unilaterally large IMT ( $R$ or $L$ ) and no large diameter & 504 & 417 & $1(0.2)$ & $7(1.7)$ \\
\hline 8) Bilaterally large diameter, no large IMT & 269 & 223 & $1(0.4)$ & $5(2.2)$ \\
\hline 9) Unilaterally large diameter, (R or L), No large IMT & 283 & 270 & $2(0.7)$ & $3(1.1)$ \\
\hline 10) No IMT or diameter large & 753 & 610 & $2(0.3)$ & $7(1.2)$ \\
\hline All & 4014 & 3262 & $66(1.6)$ & $115(3.5)$ \\
\hline \multicolumn{5}{|l|}{ Combined phenotypes } \\
\hline IMT large bilaterally, regardless of diameter (Phenotypes:1,2,5) & 1445 & 1114 & $52(3.6)$ & $73(6.6)$ \\
\hline Diameter large bilaterally, regardless of IMT (Phenotypes:1,3,8) & 1569 & 1255 & $49(3.1)$ & $75(6.0)$ \\
\hline Large IMT bilaterally and/or large diameter bilaterally (Phenotypes: 1,2,3,5,8) & 2162 & 1707 & $59(2.7)$ & $97(5.7)$ \\
\hline IMT and diameter both large on same side, unilaterally or bilaterally (Phenotypes: $1,2,3,4$ ) & 1777 & 1433 & $53(3.0)$ & $83(5.8)$ \\
\hline Large IMT, unilaterally or bilaterally, regardless of diameter (Phenotypes: 1-7) & 2709 & 2159 & $61(2.3)$ & $100(4.6)$ \\
\hline Large diameter, unilaterally or bilaterally, regardless of IMT (Phenotypes: $1,2,3,4,6,8,9)$ & 2435 & 2012 & $56(2.5)$ & $91(4.7)$ \\
\hline
\end{tabular}

*First time strokes occurring after baseline (1987-89) through December 31, 1999 with follow up of 10 to 12 years. Large is defined as $\geq$ the gender- and side-specific ultrasound medians for IMT and external diameter in Table 1. CCA common carotid artery, IMT intima-media thickness. 


\begin{tabular}{|c|c|c|c|c|c|}
\hline Risk assessment criteria/phenotypes & $\begin{array}{l}\text { Gender } \\
\text { (\#strokes) }\end{array}$ & Sensitivity & Specificity & $\begin{array}{l}\text { Positive likelihood } \\
\text { ratio }(95 \% \mathrm{Cl})\end{array}$ & $\begin{array}{l}\text { Negative likelihood ratio } \\
(95 \% \mathrm{CI})\end{array}$ \\
\hline \multirow[t]{2}{*}{ IMT and diameter both large, bilaterally } & Men (51) & 0.44 & 0.81 & $2.28(1.84-2.84)$ & $0.69(0.59-0.81)$ \\
\hline & Women (42) & 0.64 & 0.79 & $3.10(2.56-3.76)$ & $0.46(0.33-0.63)$ \\
\hline \multirow[t]{2}{*}{ IMT large bilaterally, regardless of diameter } & Men (73) & 0.63 & 0.67 & $1.92(1.66-2.22)$ & $0.55(0.43-0.69)$ \\
\hline & Women (52) & 0.79 & 0.65 & $2.23(1.96-2.55)$ & $0.33(0.21-0.52)$ \\
\hline \multirow[t]{2}{*}{ Diameter large bilaterally, regardless of IMT } & Men (75) & 0.65 & 0.63 & $1.74(1.51-2.00)$ & $0.56(0.43-0.71)$ \\
\hline & Women (49) & 0.74 & 0.61 & $1.93(1.66-2.23)$ & $0.42(0.28-0.63)$ \\
\hline \multirow{2}{*}{$\begin{array}{l}\text { IMT and diameter both large on same side, } \\
\text { unilaterally or bilaterally }\end{array}$} & Men (83) & 0.72 & 0.57 & $1.68(1.49-1.90)$ & $0.49(0.36-0.65)$ \\
\hline & Women (53) & 0.80 & 0.56 & $1.84(1.62-2.08)$ & $0.35(0.21-0.57)$ \\
\hline \multirow{2}{*}{$\begin{array}{l}\text { Large IMT, unilaterally or bilaterally, regardless } \\
\text { of diameter }\end{array}$} & Men (100) & 0.87 & 0.35 & $1.33(1.23-1.43)$ & $0.38(0.24-0.61)$ \\
\hline & Women (61) & 0.92 & 0.33 & $1.38(1.28-1.48)$ & $0.23(0.10-0.53)$ \\
\hline \multirow{2}{*}{$\begin{array}{l}\text { Large diameter, unilaterally or bilaterally, } \\
\text { regardless of IMT }\end{array}$} & Men (91) & 0.79 & 0.39 & $1.30(1.18-1.43)$ & $0.54(0.37-0.77)$ \\
\hline & Women (56) & 0.85 & 0.40 & $1.41(1.27-1.56)$ & $0.38(0.22-0.68)$ \\
\hline \multirow[t]{2}{*}{ Large IMT bilaterally and/or large diameter bilaterally } & Men (97) & 0.84 & 0.49 & $1.65(1.51-1.80)$ & $0.32(0.21-0.49)$ \\
\hline & Women (59) & 0.89 & 0.47 & $1.68(1.54-1.83)$ & $0.23(0.11-0.46)$ \\
\hline \multirow[t]{2}{*}{ Framingham score: $\mathrm{CVD}^{\dagger}$} & Men & 0.72 & 0.81 & $3.85(3.34-4.44)$ & $0.34(0.25-0.48)$ \\
\hline & Women & 0.62 & 0.81 & $3.23(2.70-3.86)$ & $0.47(0.36-0.62)$ \\
\hline \multirow[t]{2}{*}{ Cerebrovascular score $^{\dagger}$} & Men & 0.76 & 0.81 & $4.1(3.60-4.68)$ & $0.29(0.20-0.42)$ \\
\hline & Women & 0.64 & 0.81 & $3.36(2.83-3.99)$ & $0.44(0.33-0.59)$ \\
\hline
\end{tabular}

*First time strokes occurring after baseline (1987-89) through December 31, 1999 with follow up of 10-12 years. ${ }^{\dagger}$ The likelihood ratios for the Framingham scores are based on numbers calculated from the sensitivity and specificity reported for the top quintile of the Framingham scores in the Framingham population (12).

comprises over $50 \%$ of US adults [15]. IMT and external diameter reflect the impact of multiple vascular risk factors and their interactions, treatment effects, the length of each exposure, and presence of carotid plaques. While our study has several limitations, the results indicate that bilateral measures of IMT and diameter are complementary and may help address stroke risk assessment, especially in women.

B-mode ultrasound is safe, easily administered and relatively low in cost [4]; thus, fulfilling several criteria for tools for screening asymptomatic persons $[7,17]$. We used mean IMT from the CCA far wall which is likely to be more clinically feasible than a mean including additional measurements from the bifurcation and internal carotid segments where more values were missing. Prior studies have shown measurements of mean far CCA wall IMT [33] and external diameter [38] to have acceptable repeatability. A reliability coefficient of 0.98 was found for the CCA mean arterial diameter and 0.78 for CCA maximum far wall thickness [38]. Side differences in measurement error were small [33]. Overall reliability coefficients (proportion of the between person variance over total variance) for the mean of the right and left CCA far wall IMT were calculated as 0.53 to 0.54 in a small subset with little between person variability, but was estimated to be
0.70 when the full ARIC between persons variability was considered [33]. Measurement errors usually bias results toward the null [33] and so should not have produced the large effects for the arterial geometries on the right and left. However, the discordance we found for the $50^{\text {th }}$ percentile categories on the right and left could have a component of measurement error. Consideration of both the right and left could thus improve prediction by assuring the true presence of larger parameters. However, as pointed out by Howard and co-authors who found discordance for continuous measures of rightleft carotid IMT and diameter [39], much of the discordance is likely the result of the focal nature of the systemic atherosclerotic process.

That external carotid artery diameter added to stroke risk discrimination was shown previously by using diameter as a continuous measure [25]. Extending their study we found the strongest stroke risk was associated with the combined presence of large CCA IMT and large diameter. This change bilaterally was the phenotype with the highest cumulative stroke incidence. The +LR and LR found for women with this phenotype, while not optimal, were comparable to those calculated for the reported sensitivities and specificities for the top quintiles of the Framingham scores [12]. No other phenotype had comparable specificity or $+\mathrm{LR}$. 
CHD and vulnerable plaques are important contributors to stroke $[4,6]$. We previously showed that the RCCA geometry of large IMT and large diameter was significantly associated with carotid plaques in any carotid segment [40] and with incident cardiac events [41]. Others have documented larger carotid diameters in the presence of some common types of vulnerable plaques [23] which could contribute to large IMT and large diameter's association with incident strokes. Bilateral findings could also be important because it indicates more extensive disease and extent of intra- and extra-cranial atherosclerosis was recently shown to be associated with stroke occurrence in patients undergoing coronary artery bypass grafting [42]. The substantial stroke risk seen for bilaterally large IMT in the absence of large diameter may reflect the susceptibility to hypotension that can occur with narrowing of both carotids even in the absence of vulnerable plaques. However, given the associations were stronger in women and since during the period of study, ARIC was a relatively low risk population, one must consider alternative explanations.

Polak and co-authors found that CCA interadventitial distance was independently associated with left ventricular mass and proposed this association as an explanation for the ability of external diameter to improve prediction for cardiovascular events [36]. So, an alternative explanation for bilateral arterial phenotypes' usefulness in stroke prediction may be the CCA patterns' associations with major CVD risk factors. Persons with RCCA or LCCA that had combined large IMT and large diameter had less favorable risk factor distributions than other arterial geometries. Hypertension and increasing systolic blood pressure are associated with larger arterial diameters $[21,22,36]$ and may contribute to strokes. External diameter is also larger with increasing age, smoking and presence of CHD [21,22], which are all major stroke risk factors $[2,20]$. The presence of atherosclerotic disease and risk factors increase the associated CCA external diameter size with age [35]. So, the presence of bilateral diameter enlargement may indicate major risk factors for lacunar and non-lacunar stroke. While height, age, and male gender are associated with larger arterial diameters [36], we controlled for those factors (and other risk factors) in the side-specific analyses and still found significant stroke associations for the large IMT plus large diameter geometry. This indicates carotid geometry provides information for stroke risk beyond multiple risk factors.

Our study indicates that CCA IMT and external diameter measures on the right and left are complementary in identifying stroke risk. The specificity for incident stroke was higher for the phenotype of both large IMT and large diameter on both sides at baseline than that for the other phenotypes resulting in a larger probability of true positive tests to probability of false positive tests. Of the bilateral phenotypes, the low -LR for large IMT bilaterally and or large diameter bilaterally, indicates a lower probability of false negative tests to the probability of true negative tests and could help identify low risk.

Limitations of the study need to be considered. We did not optimize the cuts for IMT and diameter in our population, but used medians as a simple means for defining which parameters were large. So, our model prediction is unlikely to be overly optimistic, but might be improved with optimal cuts. The specific sets of bilateral phenotypes were not preplanned. A major limitation is that the risk stratification was not validated internally or externally nor was there any formal statistical comparison to other risk stratification method. Further, there was no assessment of whether the geometric patterns or bilateral phenotypes could be used for reclassification of risk defined by the Framingham or other stratification methods. We also did not determine whether arterial geometry was associated with stroke independently of carotid stiffness measures.

Many ARIC participants were missing information on baseline algorithm defined stroke due to a change in the questionnaire [29]. Given that participants were randomly assigned an examination date, this should not have biased our sample. We were unable to adjust for geographic location. While the follow up for stroke in our study varied from about 10 to 12 years, the variation is unlikely to have biased the sensitivity, specificity or likelihood tests because initial evaluations, where the timing varied, were selected randomly.

Atrial fibrillation is often undiagnosed in the general population [2]. So, the prediction of strokes by CCA phenotypes in our study despite not adjusting for atrial fibrillation shows the potential strength of this risk stratification method. This strength may be explained by overlap of atrial fibrillation risk factors [43] with those for combined large IMT and large diameter. We were unable to test for confounding by atrial fibrillation in our study.

\section{Conclusion}

Despite limitations, this study provides evidence that bilateral CCA patterns may be useful in identifying groups at high and low risk of stroke. However, our findings need to be confirmed in other populations and expanded to assess risk stratification improvement.

\section{Competing interests}

The authors have no conflict of interest to report.

\section{Authors' contributions}

MLE (the corresponding author) conceived and designed the study (with input), performed many statistical analyses (with input), drafted the manuscript and provided multiple revisions. GWE provided input into the design of the study and into statistical analyses, provided input into revisions 
and knowledge of ARIC ultrasound studies. KMR provided input into multiple revisions and epidemiologic concepts, ARIC procedures, and multiple revisions. ZB performed some statistical analyses, provided input into statistical design and revisions of the manuscript. RET provided input into multiple revisions and important pathologic concepts potentially impacting associations. JLM provided cardiovascular disease knowledge and input into multiple revisions and into the final manuscript. DJC provided insight into statistical issues, input into multiple revisions and expertise on the ARIC data. All authors have read and approved submission of this manuscript.

\section{Acknowledgements}

The Atherosclerosis Risk in Community (ARIC) Study is conducted and supported by NHLBI in collaboration with ARIC Study Investigators. This manuscript was prepared using the ARICLAD and so did not go through the ARIC Study manuscript approval process; and thus, does not necessarily reflect the opinions or views of the entire ARIC Study or NHLBI. Access to the data ended and so additional follow-up of more recent events are not included.

This study was funded by NHLBI grant number R21 HL076833-02.

\section{Author details}

${ }^{1}$ College of Medicine and Fay W. Boozman College of Public Health, University of Arkansas for Medical Sciences, Little Rock, AR, USA. ${ }^{2}$ Division of Public Health Sciences, Wake Forest School of Medicine, Winston-Salem, NC, USA. ${ }^{3}$ Department of Epidemiology, Gillings School of Global Public Health,

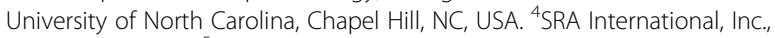
Durham, NC, USA. ${ }^{5}$ Department of Biostatistics, Fay W. Boozman College of Public Health, University of Arkansas for Medical Sciences, Little Rock, AR, USA. ${ }^{6}$ Department of Pathology, Louisiana State University Health Science Center, New Orleans, LA, USA. ${ }^{7}$ Departments of Medicine, Physiology, and Biophysics, University of Arkansas for Medical Sciences, Little Rock, AR, USA. ${ }^{8}$ Department of Biostatistics, Gillings School of Global Public Health, University of North Carolina, Chapel Hill, NC, USA.

Received: 3 May 2013 Accepted: 1 June 2013

Published: 15 June 2013

\section{References}

1. Marsh JD, Keyrouz SG: Stroke prevention and treatment. J Am Coll Cardio 2010, 56:683-691

2. Roger VL, Go AS, Lloyd-Jones DM, Benjamin EJ, Berry JD, Borden WB, Bravata DM, Dai S, Ford ES, Fox CS, Fullerton HJ, Gillespie C, Hailpern SM Heit JA, Howard VJ, Kissela BM, Kittner SJ, Lackland DT, Lichtman JH, Lisabeth LD, Makuc DM, Marcus GM, Marelli A, Matchar DB, Moy CS, Mozaffarian D, Mussolino ME, Nichol G, Paynter NP, Soliman EZ, et al: Heart disease and stroke statistics--2012 update: a report from the American Heart Association. Circulation 2012, 125:e2-e220. http://m.circ.ahajournals. org/content/125/1/e2.full.

3. Wolff T, Guirguis-Blake J, Miller T, Gillespie M, Harris R: Screening for carotid artery stenosis: an update of the evidence for the U.S. preventive services task force. Ann Intern Med 2007, 147:860-870.

4. Kwee RM, van Oostenbrugge RJ, Hofstra L, Teule GJ, van Engelshoven JMA Mess WH, Kooi ME: Identifying vulnerable carotid plaques by noninvasive imaging. Neurology 2008, 70:2401-2409.

5. Saam T, Underhill HR, Chu B, Takaya N, Cai J, Polissar NL, Yuan C, Hatsukami TS: Prevalence of American Heart Association type VI carotid atherosclerotic lesions identified by magnetic resonance imaging for different levels of stenosis as measured by duplex ultrasound. J Am Coll Cardiol 2008, 51:1014-1021.

6. Freilinger TM, Schindler A, Schmidt C, Grimm J, Cyran C, Schwarz F, Bamberg F, Linn J, Reiser M, Yuan C, Nikolaou K, Dichgans M, Saam T: Prevalence of nonstenosing, complicated atherosclerotic plaques in cryptogenic stroke. J Am Coll Cardiol Img 2012, 5:397-405.

7. Wasserman BA, Wityk RJ, Trout HH 3rd, Virmani R: Low-grade carotid stenosis: looking beyond the lumen with MRI. Stroke 2005, 36:2504-2513.

8. Berger JS, Jordan CO, Lloyd-Jones D, Blumenthal RS: Screening for cardiovascular risk in asymptomatic patients. J Am Coll Cardiol 2010, 55:1169-1177.

9. Cooney MT, Dudina A, D'Agostino R, Graham IM: Cardiovascular risk-estimation systems in primary prevention: do they differ? Do they make a difference? Can we see the future? Circulation 2010, 122:300-310.
10. Greenland P, Alpert JS, Beller GA, Benjamin EJ, Budoff MJ, Fayad ZA, Foster E, Hlatky MA, Hodgson JMB, Kushner FG, Lauer MS, Shaw LJ, Smith SC, Taylor AJ, Weintraub WS, Wenger NK: ACCF/AHA guideline for assessment of cardiovascular risk in asymptomatic adults: a report of the American College of Cardiology Foundation/American Heart Association Task Force on Practice Guidelines. J Am Coll Cardiol 2010, 56:e50-e103. http://dx.doi.org/10.1016/j.jacc.2010.09.001.

11. Chambless LE, Heiss G, Shahar E, Earp MJ, Toole J: Prediction of ischemic stroke risk in the Atherosclerosis Risk in Communities Study. Am J Epidemiol 2004, 160:259-269.

12. D'Agostino RB Sr, Vasan RS, Pencina MJ, Wolf PA, Cobain M, Massaro JM, Kannel WB: General cardiovascular risk profile for use in primary care: the Framingham Heart Study. Circulation 2008, 117:743-753.

13. Berry JD, Willis B, Gupta S, Barlow CE, Lakoski SG, Khera A, Rohatgi A, de Lemos JA RA, Haskell W, Lloyd-Jones DM: Lifetime risks for cardiovascular disease mortality by cardiorespiratory fitness levels measured at ages 45, 55, and 65 years in men. The Cooper Center Longitudinal Study. J Am Coll Cardiol 2011, 57:1604-1610.

14. Marma AK, Lloyd-Jones DM: Systematic examination of the updated Framingham heart study general cardiovascular risk profile. Circulation 2009, 120:384-390.

15. Lloyd-Jones DM: Cardiovascular risk prediction: basic concepts, current status, and future directions. Circulation 2010, 121:1768-1777.

16. Stein JH, Korcarz CE, Hurst RT, Lonn E, Kendall CB, Mohler ER, Najjar SS, Rembold CM, Post WS: Use of carotid ultrasound to identify subclinical vascular disease and evaluate cardiovascular disease risk: a consensus statement from the American Society of Echocardiography Carotid Intima-Media Thickness Task Force. J Am Soc Echocard 2008, 21:93-111.

17. Kullo IJ, Malik AR: Arterial ultrasonography and tonometry as adjuncts to cardiovascular risk stratification. J Am Coll Cardiol 2007, 49:1413-1426.

18. Polak JF, Pencina MJ, Pencina KM, O'Donnell CJ, Wolf PA, D'Agostino RB Sr: Carotid-wall intima-media thickness and cardiovascular events. N Engl J Med 2011, 365:213-221.

19. Yang EY, Chambless L, Sharrett AR, Virani SS, Liu X, Tang Z, Boerwinkle E, Ballantyne CM, Nambi V: Carotid arterial wall characteristics are associated wtih incident ischemic stroke but not coronary heart disease in the Atherosclerosis Risk in Communities (ARIC) Study. Stroke 2012, 43:103-108.

20. Ohira T, Shahar E, Chambless LE, Rosamond WD, Mosley TH Jr, Folsom AR: Risk factors for ischemic stroke subtypes: the Atherosclerosis Risk in Communities study. Stroke 2006, 37:2493-2498.

21. Crouse JR, Goldbourt U, Evans G, Pinsky J, Sharrett AR, Sorlie P, Riley W, Heiss G: for the ARIC Investigators. Risk factors and segment-specific carotid arterial enlargement in the Atherosclerosis Risk in Communities (ARIC) cohort. Stroke 1996, 27:69-75.

22. Empana JP, Zureik M, Gariepy J, Courbon D, Dartigues JF, Ritchie K, Tzourio C, Alperovitch A, Ducimetiere P: The metabolic syndrome and the carotid artery structure in noninstitutionalized elderly subjects: the Three-City Study. Stroke 2007, 38:893-899.

23. Burke AP, Kolodgie FD, Farb A, Weber D, Virmani R: Morphological predictors of arterial remodeling in coronary atherosclerosis. Circulation 2002, 105:297-303.

24. Bai $\mathrm{CH}$, Chen JR, Chiu HC, Pan WH: Lower blood flow velocity, higher resistance index, and larger diameter of extracranial carotid arteries are associated with ischemic stroke independently of carotid atherosclerosis and cardiovascular risk factors. J Clin Ultrasound 2007, 35:322-330.

25. Baldassarre D, Hamsten A, Veglia F, de Faire U, Humphries SE, Smit AJ, Giral P, Kurl S, Rauramaa R, Mannarino E, Grossi E, Paoletti R, Tremoli E, on behalf of the IMPROVE Study Group: Measurements of carotid intima-media thickness and of interadventitia common carotid diameter improve prediction of cardiovascular events. J Am Coll Cardiol 2012, 60(16):1489-1499.

26. Chatzizisis YS, Coskun AU, Jonas M, Edelman ER, Feldman CL, Stone PH: Role of endothelial shear stress in the natural history of coronary atherosclerosis and vascular remodeling: molecular, cellular, and vascular behavior. J Am Coll Cardiol 2007, 49:2379-2393.

27. Scuteri A, Chen CH, Yin FC, Chih-Tai T, Spurgeon HA, Lakatta EG: Functional correlates of central arterial geometric phenotypes. Hypertension 2001, 38:1471-1475.

28. ARIC Investigators: The Atherosclerosis Risk in Communities (ARIC) Study: design and objectives. Am J Epidemiol 1989, 129:687-702.

29. Toole JF, Lefkowitz DS, Chambless LE, Wijinberg L, Paton CC, Heiss G: Self-reported transient ischemic attack and stroke symptoms: methods and baseline prevalence. Am J Epidemiol 1996, 144:849-856. 
30. Rosamond WD, Folsom AR, Chambless LE, Wang CH, McGovern PG, Howard G, Copper LS, Shahar E: Stroke incidence and survival among middle-aged adults: 9-year follow-up of the Atherosclerosis Risk in Communities (ARIC) cohort. Stroke 1999, 30:736-743.

31. NHLBI: Atherosclerosis Risk in Communities (ARIC) Study. Operations Manual no. 6 A and B. Version 1.0, Ultrasound Assessment; Scanning and Reading Procedures. Chapel Hill, NC: ARIC Coordinating Center, School of Public Health, University of North Carolina; 1987. http://www2.cscc.unc.edu/aric/ sites/default/files/public/manuals/Ultrasound_Assessment_Scanning_Procedures.1_6a.pdf.

32. Hunt KJ, Pankow JS, Offenbacher S, Kritchevsky SB, Duncan BB, Shahar E, Sharrett AR, Heiss G: B-mode ultrasound-detected carotid artery lesions with and without acoustic shadowing and their association with markers of inflammation and endothelial activation: the Atherosclerosis Risk in Communities Study. Atherosclerosis 2002, 162:145-155.

33. Chambless LE, Zhong MM, Arnett D, Folsom AR, Riley WA, Heiss G: Variability in B-Mode ultrasound measurements in the Atherosclerosis Risk in Communities (ARIC) Study. Ultrasound Med Biol 1996, 22:545-554.

34. Lowry R: Clinical Calculator 1. Vassar College; 2001-2013. Varssarstat; website for Statistical Computation www.vassarstats.net/clin1.html accessed 2013.

35. Eigenbrodt ML, Bursac Z, Rose KM, Couper DJ, Tracy RE, Evans GW, Brancati

FL, Mehta JL: Common carotid arterial interadventitial distance (diameter) as an indicator of the damaging effects of age and atherosclerosis, a cross-sectional study of the Atherosclerosis Risk in Community Cohort Limited Access Data (ARICLAD), 1987-89. CardiovasC Ultrasound 2006, 4:1-10. http://www.cardiovascularultrasound.com/content/ $4 / 1 / 1$

36. Polak JF, Wong Q, Johnson WC, Bluemke DA, Harrington A, O'Leary DH, Yanez ND: Associations of cardiovascular risk factors, carotid intima-media thickness and left ventricular mass with inter-adventitial diameters of the common carotid artery: the Multi-Ethnic Study of Atherosclerosis (MESA). Atherosclerosis 2011, 218:344-349.

37. Allison P (Ed): Survival Analysis Using the SAS System: a Practical Guide. 1st edition. Cary, NC: SAS Institute Inc; 1995.

38. Riley WA, Barnes RW, Bond MG, Evans G, Chambless LE, Heiss G: High-resolution B-Mode ultrasound reading methods in the Atherosclerosis Risk in Communities (ARIC) Cohort. J Neuroimag 1991, 1:168-172.

39. Howard G, Sharrett AR, Heiss G, Evans GW, Chambless LE, Riley WA, Burke GL: Carotid artery intimal-medial thickness distribution in general populations as evaluated by B-Mode ultrasound. Stroke 1993, 24:1297-1304

40. Eigenbrodt ML, Bursac Z, Tracy RE, Mehta JL, Rose KM, Couper DJ: B-mode ultrasound common carotid artery intima-media thickness and external diameter: cross-sectional and longitudinal associations with carotid atherosclerosis in a large population sample. Cardiovasc Ultrasound 2008, 6:10. http://www.cardiovascularultrasound.com/content/6/1/10.

41. Eigenbrodt ML, Sukhija R, Rose KM, Tracy RE, Couper DJ, Evans GW, Bursac Z, Mehta JL: Common carotid artery wall thickness and external diameter as predictors of prevalent and incident cardiac events in a large population study. Cardiovasc Ultrasound 2007, 5:11. http://www.cardiovascularultrasound. com/content/5/1/11].

42. Lee EJ, Choi KH, Ryu JS, Jeon SB, Lee SW, Park SW, Park SJ, Lee JW, Choo SJ, Chung CH, Jung SH, Kang DW, Kim JS, Kwon SU: Stroke risk after coronary artery bypass graft surgery and extent of cerebral artery atherosclerosis. J Am Coll Cardiol 2011, 57:1811-1818.

43. Chamberlain AM, Agarwal SK, Folsom AR, Soliman EZ, Chambless LE, Crow R, Ambrose M, Alonso A: A clinical risk score for atrial fibrillation in a biracial prospective cohort (from the Atherosclerosis Risk in Communities [ARIC] study). Am J Cardiol 2011, 107:85-91.

doi:10.1186/1476-7120-11-22

Cite this article as: Eigenbrodt et al:: Bilateral common carotid artery ultrasound for prediction of incident strokes using intima-media thickness and external diameter: an observational study. Cardiovascular Ultrasound 2013 11:22.

\section{Submit your next manuscript to BioMed Central and take full advantage of:}

- Convenient online submission

- Thorough peer review

- No space constraints or color figure charges

- Immediate publication on acceptance

- Inclusion in PubMed, CAS, Scopus and Google Scholar

- Research which is freely available for redistribution

Submit your manuscript at www.biomedcentral.com/submit
C Biomed Central 